\title{
Changes in inflammation and oxidative stress signalling pathways in coarcted aorta triggered by bicuspid aortic valve and growth in young children
}

\author{
KATIE L. SKEFFINGTON ${ }^{1}$, ANDREW R. BOND ${ }^{1}$, M. GIULIA BIGOTTI ${ }^{1}$, \\ SAFA ABDULGHANI ${ }^{1,2}$, DOMINGA IACOBAZZI ${ }^{1}$, SOK-LENG KANG ${ }^{3}$, \\ KATE J. HEESOM ${ }^{4}$, MARIEANGELA C. WILSON ${ }^{4}$, SERBAN STOICA ${ }^{3}$, ROBIN MARTIN ${ }^{3}$, \\ MASSIMO CAPUTO $^{1,3}$, M. SAADEH SULEIMAN ${ }^{1}$ and MOHAMED T. GHORBEL ${ }^{1}$ \\ ${ }^{1}$ Bristol Heart Institute, Research Floor Level 7, Bristol Royal Infirmary, Bristol BS2 8HW; \\ ${ }^{2}$ Department of Congenital Heart Disease, Bristol Children's Hospital, Bristol BS2 8JB, UK; \\ ${ }^{3}$ Department of Physiology, Faculty of Medicine, Al-Quds University, P.O Box 89, Abu Dis, \\ Palestine; ${ }^{4}$ Proteomics Facility, University of Bristol, Bristol BS8 1RJ, UK
}

Received February 14, 2020; Accepted June 24, 2020

DOI: $10.3892 / \mathrm{etm} .2020 .9171$

\begin{abstract}
Neonates with coarctation of the aorta (CoA) combined with a bicuspid aortic valve (BAV) show significant structural differences compared to neonatal CoA patients with a normal tricuspid aortic valve (TAV). These effects are likely to change over time in response to growth. This study investigated proteomic differences between coarcted aortic tissue of BAV and TAV patients in children older than one month. Aortic tissue just proximal to the coarctation site was collected from 10 children $(\mathrm{BAV} ; \mathrm{n}=6,1.9 \pm 1.7$ years, TAV; $\mathrm{n}=4,1.7 \pm 1.5$ years, (mean $\pm \mathrm{SEM}, \mathrm{P}=0.92$.) Tissue were snap frozen, proteins extracted, and the extracts used for proteomic and phosphoproteomic analysis using Tandem Mass Tag (TMT) analysis. A total of 1811 protein and 76 phosphoprotein accession numbers were detected, of which 40 proteins and 6 phosphoproteins were significantly differentially expressed between BAV and TAV patients. Several canonical pathways involved in inflammation demonstrated enriched protein expression, including acute phase response signalling, EIF2 signalling and macrophage production of IL12 and reactive oxygen species. Acute phase response signalling also demonstrated enriched phosphoprotein expression, as did Th17 activation. Other pathways with significantly enriched protein expression include degradation of superoxide radicals and several pathways involved in apoptosis. This work suggests that BAV CoA patients older than one month have
\end{abstract}

Correspondence to: Professor M. Saadeh Suleiman, Bristol Heart Institute, Research Floor Level 7, Bristol Royal Infirmary, Upper Maudlin Street, Bristol BS2 8HW, UK

E-mail: m.s.suleiman@bristol.ac.uk

Key words: aortic coarctation, bicuspid aortic valve, congenital heart disease an altered proteome consistent with changes in inflammation, apoptosis and oxidative stress compared to TAV CoA patients of the same age. There is no evidence of structural differences, suggesting the pathology associated with BAV evolves with age in paediatric CoA patients.

\section{Introduction}

One of the most common congenital cardiac abnormalities is coarctation of the aorta $(\mathrm{CoA})(1)$. Around $85 \%$ of $\mathrm{CoA}$ patients also have a bicuspid aortic valve (BAV) (2). Following CoA repair, patients are at increased risk of developing cardiovascular complications including hypertension, impaired left ventricular function, aortic aneurysms and aortic dissection (3-5). Several studies have demonstrated that the risks are greater in CoA patients with BAV compared to CoA patients with the normal tricuspid valve (TAV) (6-8). The co-existence of BAV and CoA is known to alter aortic blood flow haemodynamics, and this may underlie the increased susceptibility of CoA patients with BAV to cardiovascular complications $(9,10)$.

A recent study by our group was the first to investigate differences in the vascular proteome of CoA patients with and without BAV (11). This study focused on neonatal patients (less than 3 weeks old) and demonstrated that the presence of BAV in neonatal CoA patients is associated with altered expression of proteins involved in elastin fibre formation and oxidative stress. In older CoA patients, there will have been more time for aortic remodelling to occur in response to the altered blood flow haemodynamics, and this will likely effect protein expression. Therefore, molecular changes in the coarctation area will be influenced not just by the valve and blood flow haemodynamics, but also the effects of growth. The current study therefore compares the proteome of CoA patients with and without BAV in paediatric patients older than one month, thus providing a unique insight into how the proteomic changes associated with BAV evolve in an older group of CoA patients. 


\section{Materials and methods}

Patients and sample collection. Tissue was collected from paediatric patients older than one month undergoing congenital surgery including repair of an aortic coarctation. Tissue was collected just proximal to the coarctation site. The study was conducted in accordance with the declaration of Helsinki, and the protocol was approved by the North Somerset and South Bristol Research Ethics Committee (Health Research Authority, Whitefriars, Level 3 Block B, Lewins Mead, Bristol, BS1 2NT, REC 07/H0106/172). Full informed consent was obtained from parents prior to admission for operation. The tissue was snap frozen in liquid nitrogen before being stored at $-80^{\circ} \mathrm{C}$ (BAV: $n=6$, patient age $1.9 \pm 1.7$ years (mean \pm SEM). TAV: $n=4$, patient age $1.7 \pm 1.5$ years).

Sample preparation. Proteins were extracted in radioimmuno-precipitation assay buffer (RIPA; $1 \%$ Nonidet P-40, $0.5 \%$ sodium deoxycholate, $0.1 \%$ SDS, in PBS with phosphatase and protease inhibitors), and quantified using Bradford's assay. Aliquots of $100 \mu \mathrm{g}$ were digested $\left(2.5 \mu \mathrm{g}\right.$ trypsin, $37^{\circ} \mathrm{C}$, overnight), labelled with Tandem Mass Tag (TMT) 10Plex reagents (Thermo Fisher Scientific, Loughborough, UK) and the labelled samples pooled. For total proteome analysis, aliquots of $50 \mu \mathrm{g}$ of the pooled sample were dried, re-suspended in buffer A (20 mM ammonium hydroxide, $\mathrm{pH} 10)$ and fractionated by high $\mathrm{pH}$ reversed-phase (RP) chromatography (UltiMate 3000 liquid chromatography system (Thermo Fisher Scientific) with XBridge BEH C18 Column (130 A, $3.5 \mu \mathrm{m}, 2.1 \times 150 \mathrm{~mm}$, Waters, UK). The samples were loaded in buffer $\mathrm{A}$ and peptides eluted with an increasing gradient of buffer $\mathrm{B}(20 \mathrm{mM}$ ammonium hydroxide in acetonitrile, $\mathrm{pH} 10$, $0-95 \%$ over $60 \mathrm{~min})$. The resulting fractions were dried and re-suspended in $1 \%(\mathrm{v} / \mathrm{v})$ formic acid. The remainder of the TMT-labelled pooled sample was dried and enriched using a $\mathrm{TiO}_{2}$ based phosphopeptide enrichment protocol (Pierce), before further drying and re-suspension in $1 \%(\mathrm{v} / \mathrm{v})$ formic acid.

Nano-LC mass spectrometry. Mass spectrometry was performed using an Ultimate 3000 nano-HPLC system in line with an Orbitrap Fusion Tribrid mass spectrometer (Thermo Scientific). The samples were injected onto an Acclaim PepMap C18 nano-trap column (Thermo Scientific), washed $(0.5 \% \mathrm{v} / \mathrm{v}$ acetonitrile, $0.1 \% \mathrm{v} / \mathrm{v}$ formic acid) and resolved on a $250 \mathrm{~mm}$ x $75 \mu \mathrm{m}$ Acclaim PepMap C18 reverse phase analytical column (Thermo Scientific) over an organic gradient $(150 \mathrm{~min}$, flow rate $300 \mathrm{nl} / \mathrm{min}$, solvent A: $0.1 \%$ formic acid, solvent B: Aqueous $80 \%$ acetonitrile in $0.1 \%$ formic acid, seven gradient segments: $1-6 \%$ B over $1 \mathrm{~min}$, $6-15 \%$ B over $58 \mathrm{~min}, 15-32 \%$ B over $58 \mathrm{~min}, 32-40 \%$ B over $5 \mathrm{~min}, 40-90 \%$ B over $1 \mathrm{~min}$, held at $90 \%$ B for $6 \mathrm{~min}$ and then reduced to $1 \% \mathrm{~B}$ over $1 \mathrm{~min}$ ). Nano-electrospray ionization was used to ionize the peptides $(2.0 \mathrm{kV}$, stainless steel emitter internal diameter $30 \mu \mathrm{m}$ (Thermo Scientific), capillary temperature $275^{\circ} \mathrm{C}$ ).

Spectra were acquired using an Orbitrap Fusion Tribrid mass spectrometer with Xcalibur 3.0 software (Thermo Scientific), operated using an SPS-MS3 workflow and data-dependent acquisition mode. For FTMS1 spectra a resolution of 120,000 was used, alongside an automatic gain control (AGC) target of 200,000 and a maximum injection time of $50 \mathrm{~ms}$. Precursors were filtered with an intensity threshold of 5000, with monoisotopic peak determination set to peptide and to include charge states 2-7. Previously interrogated precursors were excluded with a dynamic window (60s $+/-10 \mathrm{ppm})$. The MS2 precursors were isolated with a quadrupole mass filter (width of $1.2 \mathrm{~m} / \mathrm{z}$ ). ITMS2 spectra were collected (AGC target 10000, max injection time 70ms, CID collision energy 35\%). FTMS3 analysis was then performed (resolution 50,000, AGC target $50,000$, max injection time $105 \mathrm{~ms})$. Fragmentation of precursors was achieved using high-energy collision dissociation at normalised collision energy of $60 \%$. Synchronous Precursor Selection (SPS) was enabled to include up to five MS2 fragment ions in the FTMS3 scan.

Data processing and analysis. Processing and quantification of the raw data files was performed using Proteome Discoverer Software (Thermo Scientific, version 1.4). Peptide sequences were searched against the Uniprot human database (SEQUEST algorithm, peptide precursor mass tolerance $10 \mathrm{ppm}, \mathrm{MS} / \mathrm{MS}$ tolerance $0.6 \mathrm{Da}$ ). Oxidation of methionine $(+15.9949)$ was included as a variable modification, and both carbamido-methylation of cysteine (+57.0214) and the addition of the TMT mass tag $(+229.163)$ to peptide N-termini and lysine were included as fixed modifications. Phosphorylation of serine, threonine and tyrosine (+79.966) were also included as variable modifications in the phosphoproteomic analysis. Searches were performed with full tryptic digestion, allowing a maximum of one missed cleavage. The reverse database search option was enabled, and all the data filtered to satisfy false discovery rate of $5 \%$.

Proteins or phosphoproteins with more than one missing value per group were excluded from the analysis (12), as were putative uncharacterized proteins and proteins with accession numbers representing cDNA with weak similarity. Values are presented as a ratio to the internal standard (a pool of all samples) and represent the median of the measured peptide(s) for each protein. Fold change (BAV/TAV) ratios were calculated, and $\log _{2}$ (fold-change) was plotted against - $\log _{10}$ (P-value) on a volcano plot. A change in protein expression greater than $1.3 \mathrm{x}$ or less than $-1.3 \mathrm{x}$ and with $\mathrm{P}<0.05$ (Student's $\mathrm{t}$-test) was considered significant. These cut offs were chosen in accordance with other similar studies, for example (13). A heatmap was plotted using R software (version 3.5.1). QuickGo software was used to analyse gene ontology (GO) enrichment. Differentially expressed proteins and phosphoproteins were inputted into Ingenuity Pathway Analysis software (IPA version 46901286, Qiagen, Aarhus, Denmark) to determine significantly enriched canonical pathways (P-value of overlap calculated by Fisher's exact test right tailed).

\section{Results}

The age range of patients in the study was from one month to 10.5 years. The average age was not different significantly between groups (TAV; $1.7 \pm 1.5$ years, BAV; $1.9 \pm 1.7$ years, $\mathrm{P}=0.92$ ).

A total of 1811 protein accession numbers were detected, of which 40 were significantly differentially expressed 

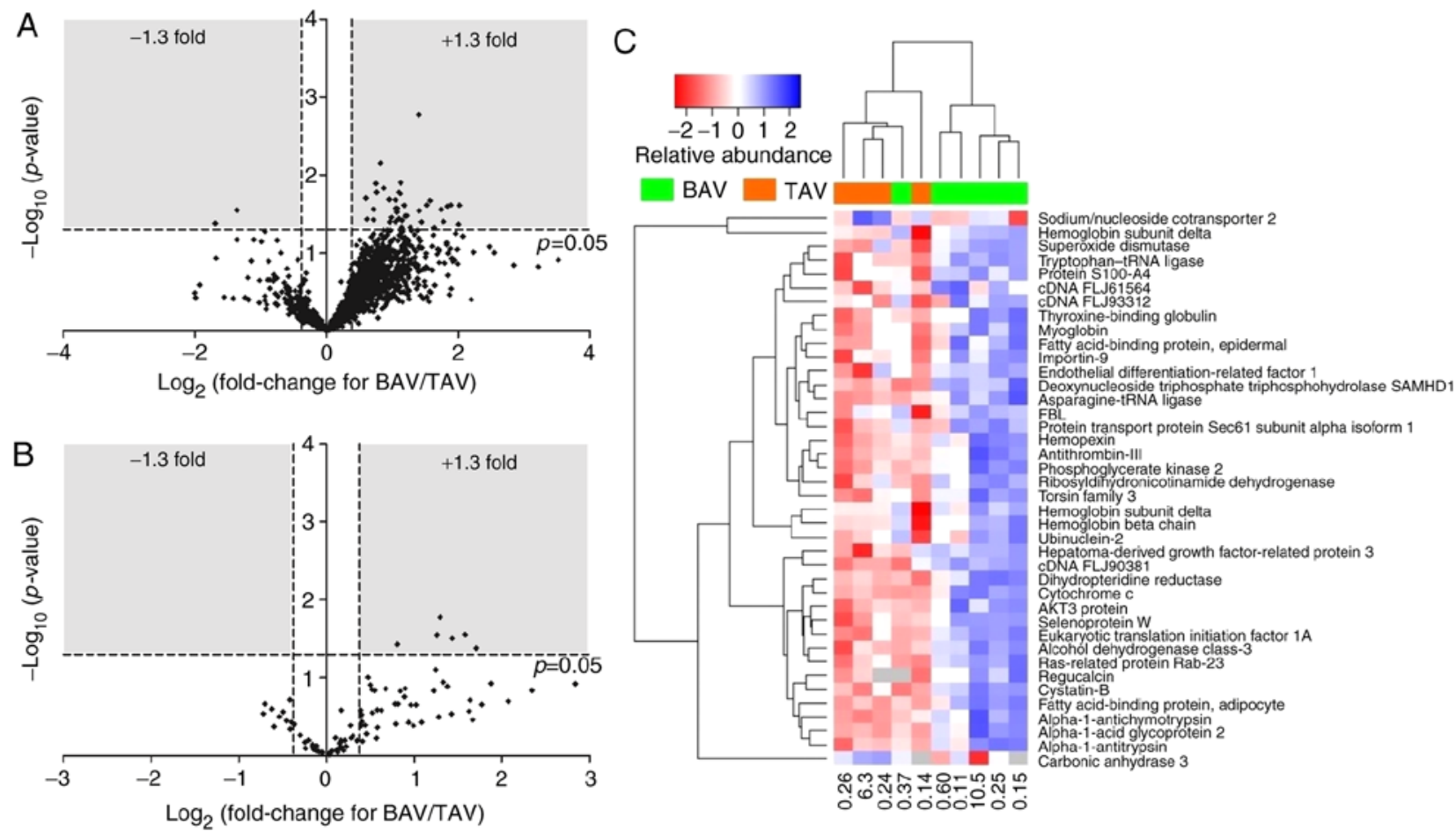

Figure 1. Differential protein expression. Volcano plots of proteins (A) and phosphoproteins (B) quantified in paediatric patients with bicuspid (BAV) vs. tricuspid (TAV) aortic valves. Each point represents the $\log _{2}$ (fold-change) between the two groups, plotted against the associated significance for the change. Proteins in the shaded area $(>1.3$ or $<0.769$ fold-change, $\mathrm{P}<0.05)$ are considered to be differentially expressed. (C) A heatmap of the 40 differentially expressed proteins. Horizontal dendrogram represents 10 independent samples. Vertical dendrogram represents the 40 proteins analysed, protein identities are listed on the right. The numbers at the bottom of each column represent the age of each patient, in years.

between BAV and TAV patients (Fig. 1A and C, Table I); 38 proteins upregulated and two proteins downregulated in BAV patients compared to TAV patients. A total of 76 phosphorylated proteins were identified, some with multiple phosphorylation sites, resulting in 92 phosphorylation site matches. 6 phosphoproteins demonstrated significantly altered expression in BAV patients compared to TAV patients; all 6 demonstrated increased phosphorylation in BAV patients (Fig. 1B, Table II).

Gene Ontology (GO) analysis was used to functionally classify the GO annotations associated with the differentially expressed proteins under the three main categories of GO analysis (molecular function, biological process and cellular component; Fig. 2). Numerous molecular functions relating to protein and ion binding were present in the top ten molecular functions, however endopeptidase and superoxide dismutase activities were also featured.

The top 20 significantly enhanced canonical pathways for protein expression are shown in Table III. The most significantly enhanced pathway was the acute phase response, a systemic response to inflammation. IPA analysis predicted activation of the acute phase response in BAV patients $(\mathrm{z}$-score $=2)$. Several other of the top 20 canonical pathways also relate to inflammation, including EIF2 signalling and macrophage production of IL12 and reactive oxygen species (ROS). Superoxide radical degradation was also highlighted as a significantly enriched canonical pathway. Finally, a number of canonical pathways involved in apoptosis, including Myc mediated apoptosis signalling, ceramide signalling and lymphotoxin $\beta$ receptor signalling, were all significantly enriched. Sixteen canonical pathways were found to be significantly enriched from the phosphoproteomic data (Table IV), including two pathways relating to inflammation, namely the acute phase response and Th17 activation.

\section{Discussion}

Our previous study (11) demonstrated that aortic tissue from neonatal CoA patients with BAV have proteomic and histological differences compared to TAV CoA patients of the same age. The changes included increased elastin content and altered expression of genes involved in elastin formation, inositol signalling and oxidative stress. The current study demonstrates that in older children (>one month) with CoA, there are still significant differences between the proteomes of BAV and TAV patients, but different proteins and pathways are involved, suggesting that the pathology associated with CoA not only develops with age, but also develops differently in BAV patients compared to TAV patients. Each group of patients contains one patient older than one year, whilst the others are aged between 1 month and one year. Interestingly, the protein expression profile of the two oldest patients are not the most extreme in either group (see Fig. 1C); this may suggest that the alterations in protein expression are similar in children between one month and ten years of age.

The presence of BAV affects the expression of proteins involved in inflammatory pathways. The most significantly enriched proteomic canonical pathway was acute phase response signalling, which was predicted to be activated in BAV patients. This 


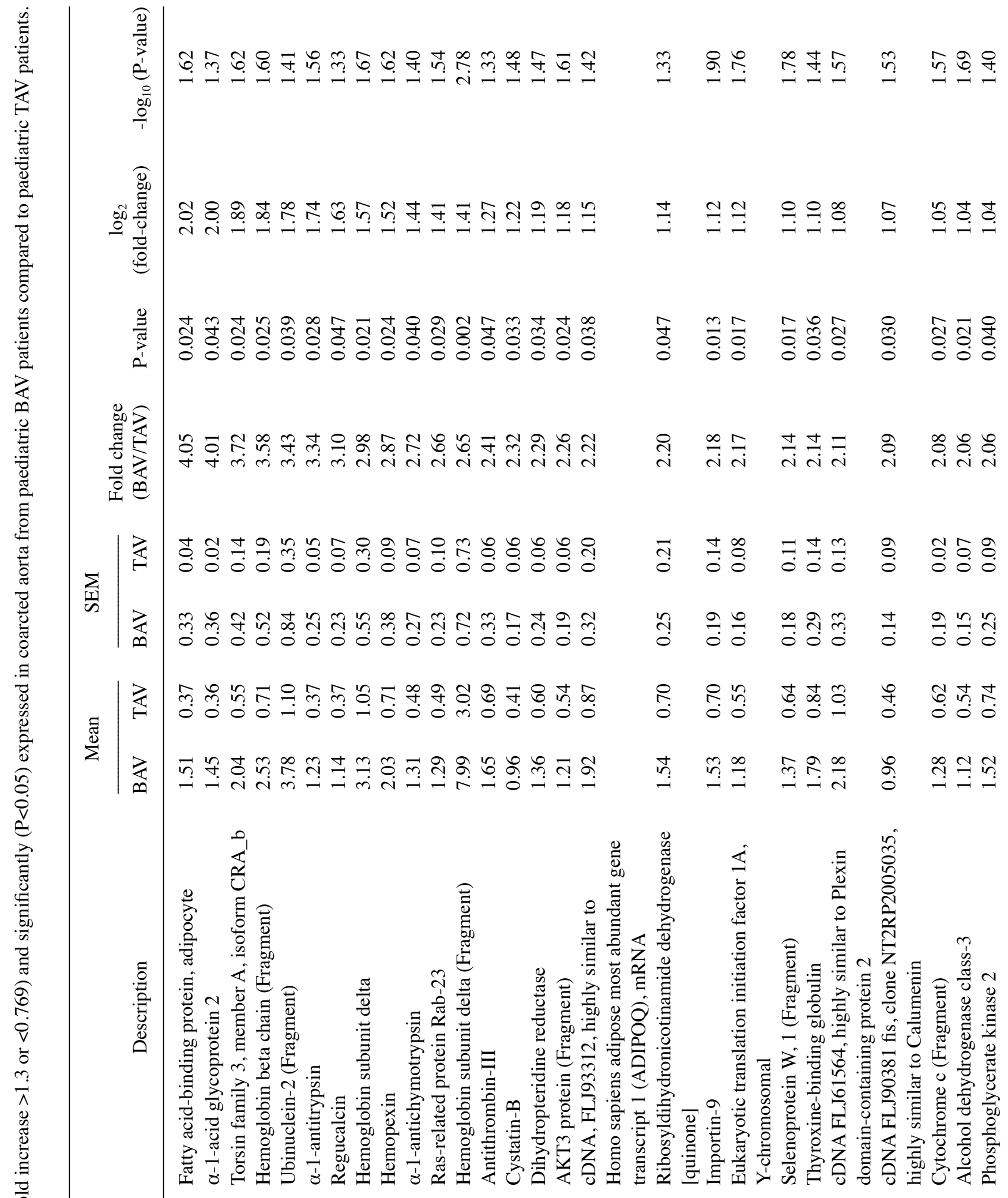

\&े

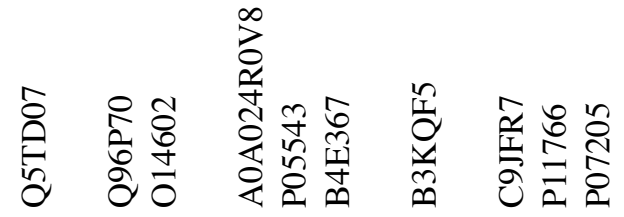



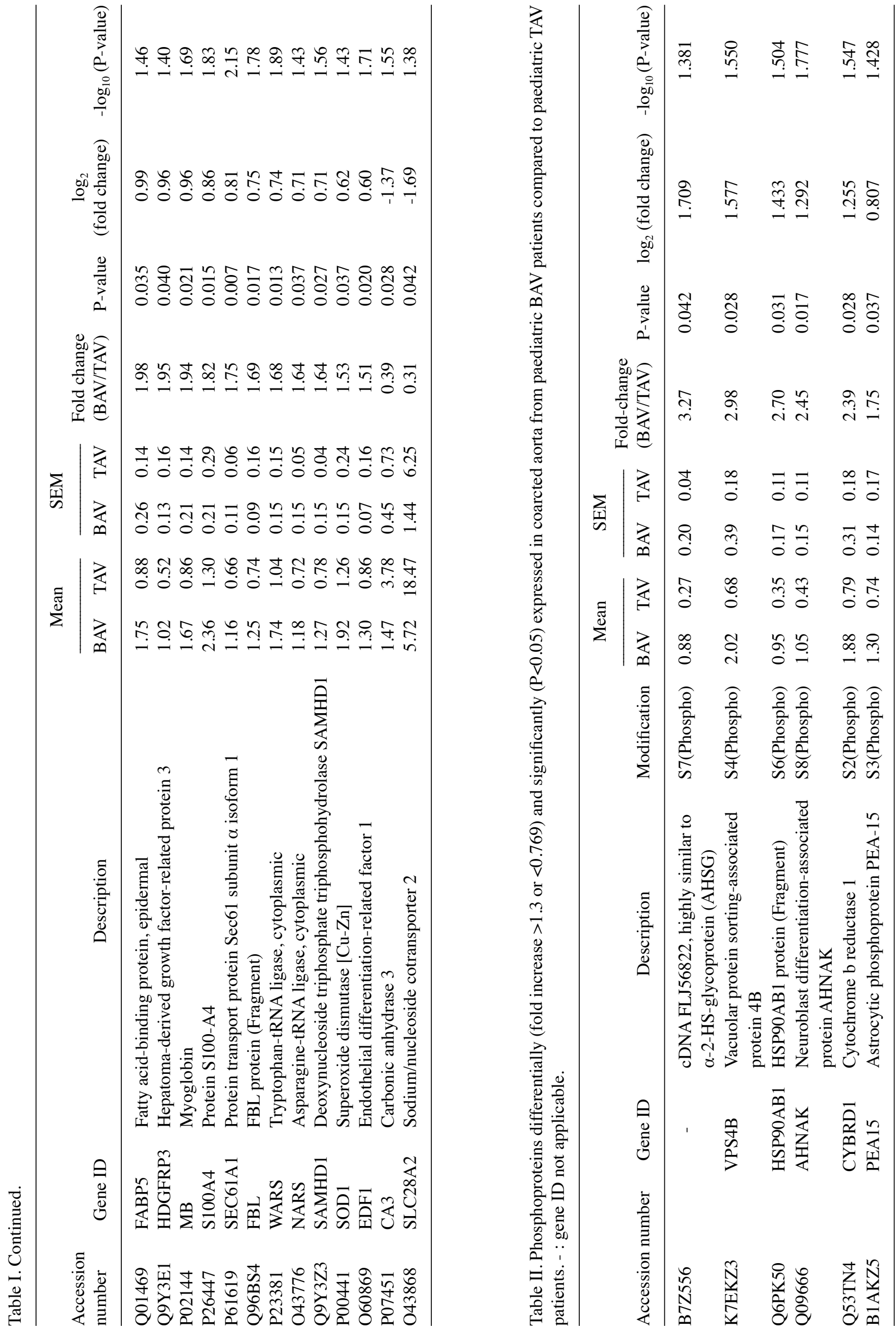


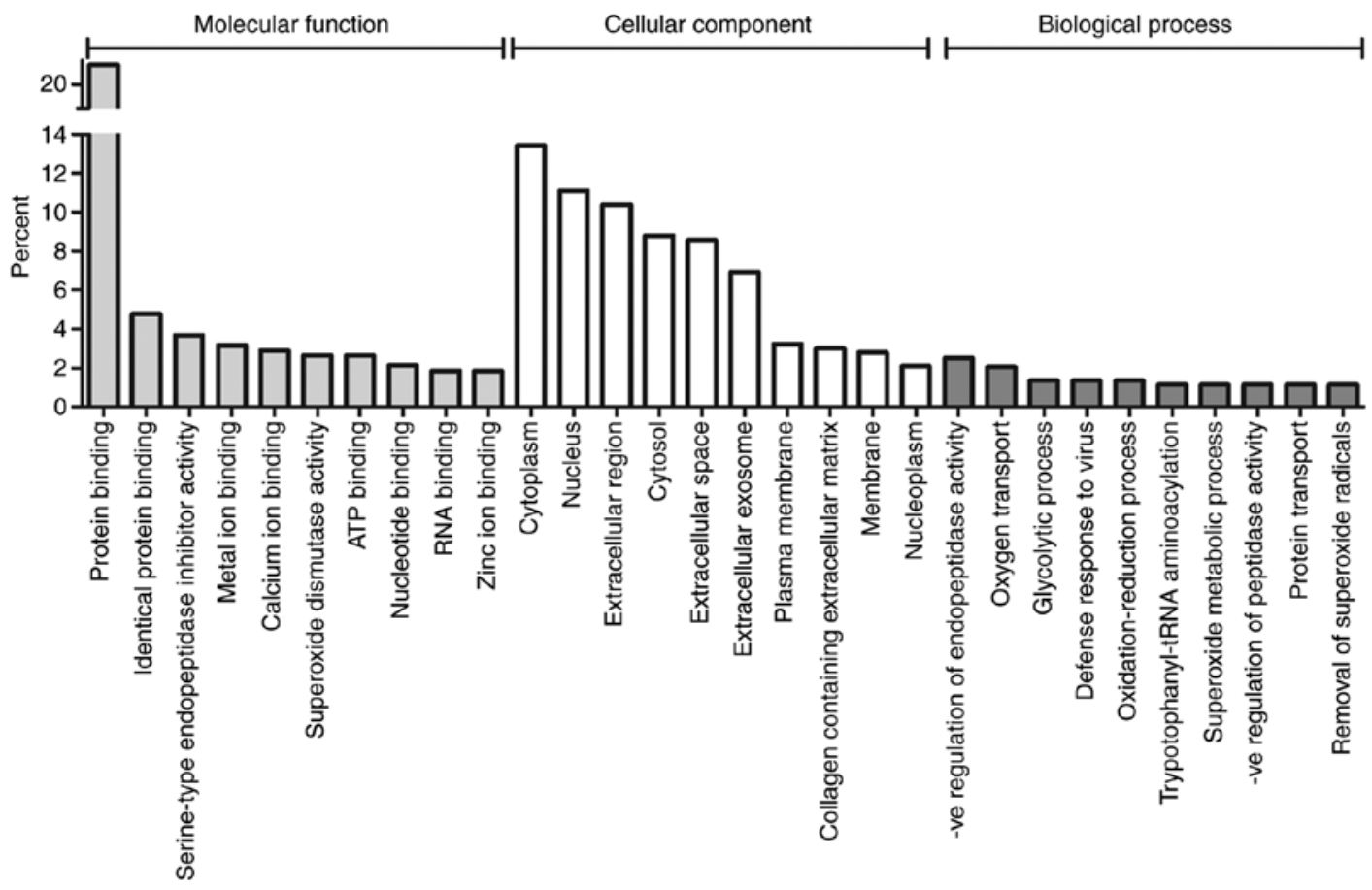

Figure 2. Functional classification by Gene Ontology (GO) analysis of proteins differentially expressed between BAV and TAV paediatric patients. The top 10 $\mathrm{GO}$ terms in each of the three main categories of GO classification (molecular function, cellular component and biological process) are displayed. The y axis represents the percentage of annotations per GO term.

pathway was also significantly enriched in the phosphoproteomics data. The acute phase response is a systemic response triggered by major local inflammation and cytokine release. Several other inflammatory canonical pathways were also significantly enriched. Within the proteomic data, production of NO, ROS and the pro-inflammatory cytokine IL-12 by macrophages were enriched, as was EIF2 signalling [a pathway involved in the regulation of pro-inflammatory cytokine expression (14)]. In the phosphoproteome analysis, the third most significant canonical pathway was activation of Th17 cells, a subset of pro-inflammatory T helper cells (15).

These changes in canonical pathways related to inflammation are driven by significant upregulation of the abundance of several proteins and phosphoproteins involved in inflammation in BAV patients compared to TAV patients. $\alpha$-1-antitrypsin (AAT), $\alpha$-1-antichymotrypsin (ACT) and $\alpha$-1-acid glycoprotein 2 (AGP) were all overexpressed in BAV patients compared to TAV patients. These proteins are all known to regulate macrophage function (16-18) and are all positive acute phase proteins $(19,20)$ (proteins whose expression increases during an inflammatory episode), as is hemopexin (21), another protein overexpressed in BAV patients. AAT and ACT both belong to the serpin family of protease inhibitors and provide negative feedback of the acute phase response via their inhibition of inflammatory cells including neutrophils and mast cells $(22,23)$. Another member of the serpin family with marked anti-inflammatory properties is anti-thrombin III (24), and this was also overexpressed in BAV patients. BAV patients also had overexpression of phosphorylated $\alpha-2$ Heremans-Schmid glycoprotein [AHSG; a negative acute phase protein $(25,26)$ ] and phosphorylated HSP90AB1 [a member of the heat shock protein 90 family, which have been demonstrated to increase secretion of pro-inflammatory cytokines (27)].
Several studies have previously suggested that differences in basal inflammation may exist between BAV and TAV patients. Local inflammation is known to occur around the abnormal BAV valve (28), however whether the presence of BAV has an effect on aortic or systemic inflammation is less clear. The combination of $\mathrm{CoA}$ and BAV has been demonstrated to cause changes in aortic blood flow haemodynamics including altered indices of shear stress (9), factors which are, in turn, known to affect vascular inflammatory pathways (29). However, studies comparing inflammatory markers such as MMPs, myeloperoxidase or measures of macrophage infiltration in BAV vs. TAV patients have mixed conclusions: Some studies found an increase in inflammatory markers associated with BAV whilst others found no difference or reduced inflammation (30-34).

The trauma of cardiac surgery is known to activate the complement system even when no cardiopulmonary bypass is involved (35). Although the full immunological response takes hours to days to materialise, markers of complement activation and some markers of inflammation start increasing measurably during surgery itself (35), and therefore some inflammatory proteomic changes are likely to already be occurring at the timepoint our samples were taken. It is possible that the differences between BAV and TAV patients in the current study indicate that BAV patients have a greater inflammatory response to surgery than TAV patients. Possibly changes in both basal immunological status and in the response to surgery exist. One recent paper found that BAV patients have decreased T and B lymphocyte levels and the authors suggested that BAV patients have 'an old immune system' which is 'more easily vulnerable to internal and external stressors' (31).

The presence of BAV affects the expression of proteins involved in oxidative stress and apoptosis. The antioxidant 
Table III. Top twenty significant canonical pathways with enriched protein expression. IPA: Ingenuity Pathway Analysis.

\begin{tabular}{|c|c|c|}
\hline IPA canonical pathway & P-value & Molecule(s) \\
\hline Acute Phase Response Signalling & $1.52 \times 10^{-5}$ & HPX, AKT3, ORM2, SERPINA1, SERPINA3 \\
\hline FXR/RXR Activation & $7.10 \times 10^{-5}$ & HPX, AKT3, ORM2, SERPINA1 \\
\hline Amyotrophic Lateral Sclerosis Signalling & $1.04 \times 10^{-3}$ & AKT3, CYCS, SOD1 \\
\hline LXR/RXR Activation & $1.27 \times 10^{-3}$ & HPX, ORM2, SERPINA1 \\
\hline Coagulation System & $1.74 \times 10^{-3}$ & SERPINC1, SERPINA1 \\
\hline Iron homeostasis signalling pathway & $1.81 \times 10^{-3}$ & HPX, HBD, HBB \\
\hline tRNA Charging & $2.16 \times 10^{-3}$ & NARS, WARS \\
\hline IL-12 Signalling and Production in Macrophages & $2.26 \times 10^{-3}$ & ORM2,AKT3, SERPINA1 \\
\hline Formaldehyde Oxidation II (Glutathione-dependent) & $3.53 \times 10^{-3}$ & ADH5 \\
\hline Docosahexaenoic Acid (DHA) Signalling & $4.10 \times 10^{-3}$ & AKT3, CYCS \\
\hline Production of Nitric Oxide and Reactive Oxygen & $5.55 \times 10^{-3}$ & ORM2, AKT3, SERPINA1 \\
\hline \multicolumn{3}{|l|}{ Species in Macrophages } \\
\hline Lymphotoxin $\beta$ Receptor Signalling & $6.61 \times 10^{-3}$ & AKT3, CYCS \\
\hline Phenylalanine Degradation I (Aerobic) & $7.04 \times 10^{-3}$ & QDPR \\
\hline EIF2 Signalling & $7.90 \times 10^{-3}$ & WARS, AKT3, EIF1AY \\
\hline Myc Mediated Apoptosis Signalling & $8.79 \times 10^{-3}$ & AKT3, CYCS \\
\hline Small Cell Lung Cancer Signalling & $1.03 \times 10^{-2}$ & AKT3, CYCS \\
\hline Superoxide Radicals Degradation & $1.40 \times 10^{-2}$ & SOD1 \\
\hline Ceramide Signalling & $1.45 \times 10^{-2}$ & AKT3, CYCS \\
\hline VEGF Signalling & $1.73 \times 10^{-2}$ & AKT3, EIF1AY \\
\hline Glucose and Glucose-1-phosphate Degradation & $1.92 \times 10^{-2}$ & RGN \\
\hline
\end{tabular}

Table IV. Significant canonical pathways with enriched phosphoprotein expression. IPA: Ingenuity Pathway Analysis.

\begin{tabular}{lll}
\hline IPA canonical pathway & P-value & Molecule \\
\hline Mitotic Roles of Polo-Like Kinase & $1.77 \times 10^{-2}$ & HSP90AB1 \\
Hypoxia Signalling in the Cardiovascular System & $1.99 \times 10^{-2}$ & HSP90AB1 \\
Th17 Activation Pathway & $2.44 \times 10^{-2}$ & HSP90AB1 \\
Prostate Cancer Signalling & $2.47 \times 10^{-2}$ & HSP90AB1 \\
Neuregulin Signalling & $2.57 \times 10^{-2}$ & HSP90AB1 \\
Nitric Oxide Signalling in the Cardiovascular System & $2.68 \times 10^{-2}$ & HSP90AB1 \\
PPAR Signalling & $2.78 \times 10^{-2}$ & HSP90AB1 \\
Telomerase Signalling & $2.89 \times 10^{-2}$ & HSP90AB1 \\
LXR/RXR Activation & $3.23 \times 10^{-2}$ & AHSG \\
FXR/RXR Activation & $3.36 \times 10^{-2}$ & AHSG \\
PI3K/AKT Signalling & $3.52 \times 10^{-2}$ & HSP90AB1 \\
Iron homeostasis signalling pathway & $3.65 \times 10^{-2}$ & CYBRD1 \\
Aryl Hydrocarbon Receptor Signalling & $3.81 \times 10^{-2}$ & HSP90AB1 \\
Aldosterone Signalling in Epithelial Cells & $4.23 \times 10^{-2}$ & HSP90AB1 \\
eNOS Signalling & $4.26 \times 10^{-2}$ & HSP90AB1 \\
Acute Phase Response Signalling & $4.75 \times 10^{-2}$ & AHSG \\
\hline
\end{tabular}

enzyme cytosolic superoxide dismutase (SOD1) was significantly overexpressed in BAV CoA patients compared to CoA patients with a normal aortic valve, and superoxide radical degradation and superoxide dismutase activity were highlighted in canonical pathway analysis and gene ontology analysis respectively (Fig. 2 and Table III). These findings suggest there may be differences in the levels of oxidative stress between BAV and TAV CoA patients. Previous studies have demonstrated that adult BAV patients have increased aortic oxidative stress compared to similar patients with a morphologically normal aortic valve (36). This may be the result of altered mechanical stretch in the aortic walls of BAV patients as vascular mechanical stretch is a known stimulant of superoxide production (37). A previous study in adults found 
increased SOD1 expression in the aorta of some subsets of BAV patients (38); to the best of our knowledge the data in the current study is the first to suggest that an association of increased SOD1 expression with BAV also exists in paediatric CoA patients. However it is interesting that in our neonatal data set (11) there is no significant difference in SOD1 expression, suggesting the change in expression may only occur in older children.

Previous studies in neonates with BAV associated with CoA and adults with BAV have demonstrated decreased expression of the extracellular form of superoxide dismutase (SOD3) associated with BAV $(11,38,39)$, however there was no significant change in SOD3 expression in the current data set. One of only two proteins to be significantly downregulated in BAV patients was however carbonic anhydrase III, which is known to have an antioxidant role under conditions of oxidative stress (40). Clearly much more work is required to understand the changes in oxidative stress and antioxidant defence mechanisms which occur at different ages in BAV patients with and without CoA.

Two proteins involved in apoptotic pathways, AKT3 and cytochrome c, were also significantly overexpressed in BAV CoA patients compared to TAV CoA patients. This resulted in the enrichment of a number of canonical pathways (Myc mediated apoptosis signalling, ceramide signalling and lymphotoxin $\beta$ receptor signalling) which are involved in apoptosis (41-43). Several previous studies have reported an increase in vascular smooth muscle cell (VSMC) apoptosis in BAV patients, and have linked this to the increased incidence of in aortic dilation and aortic aneurysms in BAV patients (44-47). The degree to which this is a genetic effect or secondary to changes in blood flow haemodynamics, or a combination of both factors, is controversial $(44,48-50)$.

Interestingly, there were also alterations in the ratio of haemoglobin chains. Various haemoglobin subunits are expressed in a number of non-erythroid cells, including lungs, neurons, endometrium and blood vessel walls $(51,52)$. In the current study there was overexpression of haemoglobins beta and delta in the aorta of BAV CoA patients compared to TAV CoA patients. The role of the beta and delta subunits in the aortic wall is unclear however it has been suggested that haemoglobin $\alpha$ acts from within blood vessel walls to help regulate nitric oxide release and vascular tone (51). It is possible therefore that the observed changes relate to the altered blood flow haemodynamics in BAV patients, however much more work is needed to understand this.

Paediatric CoA patients with BAV have a greater long-term risk of cardiovascular complications than similar patients with TAV (6-8). This study demonstrates significant differences between the proteome of the coarcted aortic tissue of young CoA patients with and without BAV, indicating that inflammation, oxidative stress and apoptotic pathways could contribute to future complications associated with BAV patients. The structural changes observed in our previous study in neonates are no longer apparent. In the long-term, improved understanding of the molecular differences between these patients coupled with an understanding of how the changes evolve with age should help to optimise treatment strategies.

Limitations of the study include the lack of tissue from a healthy control group; this would help to elucidate how the proteomic differences observed in BAV patients in this study are affected by the presence of the CoA. However, such tissues would be very difficult to obtain. Using tissues from only diseased individuals may also increase the likelihood that pathways are involved which deviate from the canonical paradigms, and these may not have been fully uncovered by our analysis. Slight regional differences in the exact location of the tissue collection may also have introduced some variation into our analyses. Additionally, validation of the changes in protein expression using western blotting would be informative, however very small sample sizes made this difficult.

\section{Acknowledgements}

Not applicable.

\section{Funding}

The present study was funded by a grant from the British Heart Foundation (grant no. CH/1/32804.)

\section{Data availability}

The datasets used and/or analyzed during the current study are available from the corresponding author on reasonable request.

\section{Author contributions}

Conceptualization: MC, MTG, MSS; investigation and analysis: KLS, AJB, SA-G, DI, KJH, MCW, MTG, MGB; sample collection: MC, RM, S-LK, SS, writing-original draft preparation: MSS, KLS; writing-review and editing: KLS, MGB, MSS, MC; funding acquisition: MTG, MC, MSS. All authors read and approved the final manuscript.

\section{Ethics approval and consent to participate}

The study was conducted in accordance with the declaration of Helsinki, and the protocol was approved by the North Somerset and South Bristol Research Ethics Committee (Health Research Authority, Whitefriars, Level 3 Block B, Lewins Mead, Bristol, BS1 2NT, REC 07/H0106/172). Full informed consent was obtained from parents prior to admission for operation.

\section{Patients consent for publication}

Not applicable.

\section{Competing interests}

The authors declare that they have no competing interests.

\section{References}

1. Teo LL, Cannell T, Babu-Narayan SV, Hughes $M$ and Mohiaddin RH: Prevalence of associated cardiovascular abnormalities in 500 patients with aortic coarctation referred for cardiovascular magnetic resonance imaging to a tertiary center. Pediatr Cardiol 32: 1120-1127, 2011. 
2. Sinning C, Zengin E, Kozlik-Feldmann R, Blankenberg S, Rickers C, von Kodolitsch Y and Girdauskas E: Bicuspid aortic valve and aortic coarctation in congenital heart diseaseimportant aspects for treatment with focus on aortic vasculopathy. Cardiovasc Diagn Ther 8: 780-788, 2018.

3. Preventza O, Livesay JJ, Cooley DA, Krajcer Z, Cheong BY and Coselli JS: Coarctation-associated aneurysms: A localized disease or diffuse aortopathy. Ann Thorac Surg 95: 1961-1967, 2013.

4. Webb G: Treatment of coarctation and late complications in the adult. Semin Thorac Cardiovasc Surg 17: 139-142, 2005.

5. Yin Z, Yang JR, Wei YS, Liang BL, Wei YB, Zhou KQ, Wang Z, Yan B and Gao YL: Ischemia-reperfusion injury in an aortic dissection patient. Am J Emerg Med 33: 987.e5-e6, 2015.

6. Bambul Heck P, Pabst von Ohain J, Kaemmerer H, Ewert P and Hager A: Survival and cardiovascular events after coarctation-repair in long-term follow-up (COAFU): Predictive value of clinical variables. Int J Cardiol 228: 347-351, 2017.

7. Jashari H, Lannering K, Ibrahimi P, Djekic D, Mellander M, Rydberg A and Henein MY: Persistent reduced myocardial deformation in neonates after CoA repair. Int J Cardiol 221: 886-891, 2016.

8. Oliver JM, Gallego P, Gonzalez A, Aroca A, Bret M and Mesa JM: Risk factors for aortic complications in adults with coarctation of the aorta. J Am Coll Cardiol 44: 1641-1647, 2004.

9. Keshavarz-Motamed Z, Garcia J and Kadem L: Fluid dynamics of coarctation of the aorta and effect of bicuspid aortic valve. PLoS One 8: e72394, 2013.

10. Pedersen TA: Late morbidity after repair of aortic coarctation. Dan Med J 59: B4436, 2012.

11. Skeffington KL, Bond AR, Abdul-Ghani S, Iacobazzi D, Kang SL, Heesom KJ, Wilson MC, Ghorbel M, Stoica S and Martin R: Bicuspid aortic valve alters aortic protein expression profile in neonatal coarctation patients. J Clin Med 8: 5172019.

12. Valikangas T, Suomi T and Elo LL: A comprehensive evaluation of popular proteomics software workflows for label-free proteome quantification and imputation. Brief Bioinform 19: $1344-1355,2018$.

13. Bond AR, Iacobazzi D, Abdul-Ghani S, Ghorbel MT, Heesom KJ, George SJ, Caputo M, Suleiman MS and Tulloh RM: The cardiac proteome in patients with congenital ventricular septal defect: A comparative study between right atria and right ventricles. J Proteomics 191: 107-113, 2019.

14. Shrestha N, Bahnan W, Wiley DJ, Barber G, Fields KA and Schesser K: Eukaryotic initiation factor 2 (eIF2) signaling regulates proinflammatory cytokine expression and bacterial invasion. J Biol Chem 287: 28738-28744, 2012.

15. Sandquist I and Kolls J: Update on regulation and effector functions of Th17. cells. F1000Res 7: 205, 2018

16. Bories PN, Guenounou M, Feger J, Kodari E, Agneray J and Durand G: Human alpha 1-acid glycoprotein-exposed macrophages release interleukin 1 inhibitory activity. Biochem Biophys Res Commun 147: 710-715, 1987.

17. Krotova K, Marek GW, Wang RL, Aslanidi G, Hoffman BE, Khodayari N, Rouhani FN and Brantly ML: Alpha-1 antitrypsindeficient macrophages have increased matriptase-mediated proteolytic activity. Am J Respir Cell Mol Biol 57: 238-247, 2017.

18. Skeel A and Leonard EJ: alpha 1-Antichymotrypsin is the human plasma inhibitor of macrophage ectoenzymes that cleave pro-macrophage stimulating protein. J Biol Chem 276: 21932-21937, 2001.

19. Fournier T, Medjoubi NN and Porquet D: Alpha-1-acid glycoprotein. Biochim Biophys Acta 1482: 157-171, 2000.

20. Jain S, Gautam V and Naseem S: Acute-phase proteins: As diagnostic tool. J Pharm Bioallied Sci 3: 118-127, 2011.

21. Rolla S, Ingoglia G, Bardina V, Silengo L, Altruda F, Novelli F and Tolosano E: Acute-phase protein hemopexin is a negative regulator of Th17 response and experimental autoimmune encephalomyelitis development. J Immunol 191: 5451-5459, 2013.

22. Janciauskiene $S$, Wrenger $S$, Immenschuh S, Olejnicka B, Greulich T, Welte T and Chorostowska-Wynimko J: The multifaceted effects of Alpha1-antitrypsin on neutrophil functions. Front Pharmacol 9: 341, 2018.

23. Kalsheker NA: Alpha 1-antichymotrypsin. Int J Biochem Cell Biol 28: 961-964, 1996.

24. Levy JH, Sniecinski RM, Welsby IJ and Levi M: Antithrombin: Anti-inflammatory properties and clinical applications. Thromb Haemost 115: 712-728, 2016.

25. Haglund AC, Ek B and Ek P: Phosphorylation of human plasma alpha2-Heremans-Schmid glycoprotein (human fetuin) in vivo. Biochem J 357: 437-445, 2001.
26. Lebreton JP, Joisel F, Raoult JP, Lannuzel B, Rogez JP and Humbert G: Serum concentration of human alpha 2 HS glycoprotein during the inflammatory process: Evidence that alpha 2 HS glycoprotein is a negative acute-phase reactant. J Clin Invest 64: $1118-1129,1979$.

27. Tukaj S, Zillikens D and Kasperkiewicz M: Inhibitory effects of heat shock protein 90 blockade on proinflammatory human Th1 and Th17 cell subpopulations. J Inflamm (Lond) 11: 10, 2014.

28. Mathieu P, Bosse Y, Huggins GS, Della Corte A, Pibarot P, Michelena HI, Limongelli G, Boulanger MC, Evangelista A, Bédard E, et al: The pathology and pathobiology of bicuspid aortic valve: State of the art and novel research perspectives. J Pathol Clin Res 1: 195-206, 2015.

29. Helderman F, Segers D, de Crom R, Hierck BP, Poelmann RE, Evans PC and Krams R: Effect of shear stress on vascular inflammation and plaque development. Curr Opin Lipidol 18: 527-533, 2007.

30. Ali OA, Chapman M, Nguyen TH, Chirkov YY, Heresztyn T, Mundisugih $\mathbf{J}$ and Horowitz JD: Interactions between inflammatory activation and endothelial dysfunction selectively modulate valve disease progression in patients with bicuspid aortic valve. Heart 100: 800-805, 2014.

31. Balistreri CR, Buffa S, Allegra A, Pisano C, Ruvolo G, Colonna-Romano G, Lio D, Mazzesi G, Schiavon S, Greco E, et al: A typical immune T/B subset profile characterizes bicuspid aortic valve: In an old status? Oxid Med Cell Longev 2018: 5879281, 2018.

32. Balistreri CR, Pisano C, Candore G, Maresi E, Codispoti M and Ruvolo G: Focus on the unique mechanisms involved in thoracic aortic aneurysm formation in bicuspid aortic valve versus tricuspid aortic valve patients: Clinical implications of a pilot study. Eur J Cardiothorac Surg 43: e180-e186, 2013.

33. LeMaire SA, Wang X, Wilks JA, Carter SA, Wen S, Won T, Leonardelli D, Anand G, Conklin LD, Wang XL, et al: Matrix metalloproteinases in ascending aortic aneurysms: bicuspid versus trileaflet aortic valves. J Surg Res 123: 40-48, 2005.

34. Tzemos N, Lyseggen E, Silversides C, Jamorski M, Tong JH, Harvey P,Floras J and Siu S: Endothelial function, carotid-femoral stiffness, and plasma matrix metalloproteinase-2 in men with bicuspid aortic valve and dilated aorta. J Am Coll Cardiol 55: 660-668, 2010

35. Gu YJ, Mariani MA, Boonstra PW, Grandjean JG and van Oeveren W: Complement activation in coronary artery bypass grafting patients without cardiopulmonary bypass: The role of tissue injury by surgical incision. Chest 116: $892-898$, 1999.

36. Billaud M, Phillippi JA, Kotlarczyk MP, Hill JC, Ellis BW, St Croix CM, Cantu-Medéllin N, Kelley EE and Gleason TG: Elevated oxidative stress in the aortic media of patients with bicuspid aortic valve. J Thorac Cardiovasc Surg 154: 1756-1762, 2017.

37. Birukov KG: Cyclic stretch, reactive oxygen species, and vascular remodeling. Antioxid Redox Signal 11: 1651-1667, 2009.

38. Phillippi JA, Hill JC, Billaud M, Green BR, Kotlarczyk MP and Gleason TG: Bicuspid aortic valve morphotype correlates with regional antioxidant gene expression profiles in the proximal ascending aorta. Ann Thorac Surg 104: 79-87, 2017.

39. Arcucci A, Ruocco MR, Albano F, Granato G, Romano V, Corso G, Bancone C, De Vendittis E, Della Corte A and Montagnani S: Analysis of extracellular superoxide dismutase and Akt in ascending aortic aneurysm with tricuspid or bicuspid aortic valve. Eur J Histochem 58: 2383, 2014.

40. Di Fiore A, Monti DM, Scaloni A, De Simone G and Monti SM: Protective role of carbonic anhydrases iii and vii in cellular defense mechanisms upon redox unbalance. Oxid Med Cell Longev 2018: 2018306, 2018.

41. Browning JL, Miatkowski K, Sizing I, Griffiths D, Zafari M, Benjamin CD, Meier W and Mackay F: Signaling through the lymphotoxin beta receptor induces the death of some adenocarcinoma tumor lines. J Exp Med 183: 867-878, 1996.

42. Haimovitz-Friedman A, Kolesnick RN and Fuks Z: Ceramide signaling in apoptosis. Br Med Bull 53: 539-553, 1997.

43. Hoffman B and Liebermann DA: Apoptotic signaling by c-MYC. Oncogene 27: 6462-6472, 2008.

44. Bonderman D, Gharehbaghi-Schnell E, Wollenek G, Maurer G, Baumgartner $\mathrm{H}$ and Lang IM: Mechanisms underlying aortic dilatation in congenital aortic valve malformation. Circulation 99: 2138-2143, 1999.

45. Cecconi M, Nistri S, Quarti A, Manfrin M, Colonna PL, Molini E and Perna GP: Aortic dilatation in patients with bicuspid aortic valve. J Cardiovasc Med (Hagerstown) 7: 11-20, 2006. 
46. Nataatmadja M, West M, West J, Summers K, Walker P, Nagata M and Watanabe T: Abnormal extracellular matrix protein transport associated with increased apoptosis of vascular smooth muscle cells in marfan syndrome and bicuspid aortic valve thoracic aortic aneurysm. Circulation 108 (Suppl 1): II329-II334, 2003.

47. Schmid FX, Bielenberg K, Schneider A, Haussler A, Keyser A and Birnbaum D: Ascending aortic aneurysm associated with bicuspid and tricuspid aortic valve: Involvement and clinical relevance of smooth muscle cell apoptosis and expression of cell deathinitiating proteins. Eur J Cardiothorac Surg 23: 537-543, 2003.

48. Hahn RT, Roman MJ, Mogtader AH and Devereux RB: Association of aortic dilation with regurgitant, stenotic and functionally normal bicuspid aortic valves. J Am Coll Cardiol 19: 283-288, 1992.

49. Harrison OJ, Visan AC, Moorjani N, Modi A, Salhiyyah K, Torrens C, Ohri S and Cagampang FR: Defective NOTCH signaling drives increased vascular smooth muscle cell apoptosis and contractile differentiation in bicuspid aortic valve aortopathy: A review of the evidence and future directions. Trends Cardiovasc Med 29: 61-68, 2019.
50. Niwa K, Perloff JK, Bhuta SM, Laks H, Drinkwater DC, Child JS and Miner PD: Structural abnormalities of great arterial walls in congenital heart disease: Light and electron microscopic analyses. Circulation 103: 393-400, 2001.

51. Butcher JT, Johnson T, Beers J, Columbus L and Isakson BE: Hemoglobin alpha in the blood vessel wall. Free Radic Biol Med 73: 136-142, 2014.

52. Saha D, Patgaonkar M, Shroff A, Ayyar K, Bashir T and Reddy KV: Hemoglobin expression in nonerythroid cells: Novel or ubiquitous? Int J Inflam 2014: 803237, 2014.

(c) (i) This work is licensed under a Creative Commons Attribution 4.0 International (CC BY 4.0) License. 